PROCEEDINGS OF THE

AMERICAN MATHEMATICAL SOCIETY

Volume 136, Number 2, February 2008, Pages 461-466

S 0002-9939(07)09029-6

Article electronically published on October 24, 2007

\title{
THE TORSION OF $p$-RAMIFIED IWASAWA MODULES II
}

\author{
SATOSHI FUJII
}

(Communicated by Ken Ono)

\begin{abstract}
In this article we prove the existence of a non-trivial torsion of the 3-ramified Iwasawa mocule over the $\mathbb{Z}_{3}^{2}$-extension of an imaginary quadratic field.
\end{abstract}

\section{INTRODUCTION}

Let $p$ be a prime number, $k / \mathbb{Q}$ a finite extension of the rational number field $\mathbb{Q}$ and $\widetilde{k}$ the compositum of all $\mathbb{Z}_{p}$-extensions of $k$, where $\mathbb{Z}_{p}$ is the additive group of $p$-adic integers. Then one has

$$
\operatorname{Gal}(\widetilde{k} / k) \simeq \mathbb{Z}_{p}^{\oplus r_{2}(k)+1}
$$

if $k / \mathbb{Q}$ is abelian, where $r_{2}(k)$ denotes the number of complex primes of $k$ (see Corollary 5.32 and Theorem 13.4 of [17]). Let $M_{\widetilde{k}} / \widetilde{k}$ be the maximal pro- $p$ abelian extension which is unramified outside all primes lying above $p$ and $\infty$, and $X_{\widetilde{k}}=\operatorname{Gal}\left(M_{\widetilde{k}} / \widetilde{k}\right)$ denotes its Galois group. The module $X_{\widetilde{k}}$ has natural $\Lambda_{\widetilde{k} / k}=\mathbb{Z}_{p}[[\operatorname{Gal}(\widetilde{k} / k)]]$-module structure, and this subject had been deeply studied by Greenberg [5, Jannsen [10 and Nguyen Quang Do [15] including the case where $k$ is a local field.

The author asked in a previous article [4] whether the torsion submodule $\operatorname{Tor}_{\Lambda_{\tilde{k} / k}} X_{\widetilde{k}}$ of $X_{\widetilde{k}}$ over $\Lambda_{\widetilde{k} / k}$ is always trivial or not when $k$ is not a totally real field and then obtained partial results for imaginary quadratic fields as follows:

Theorem A. (1) For any prime number $p$, there exist infinitely many imaginary quadratic fields $k$ such that $\operatorname{Tor}_{\Lambda_{\tilde{k} / k}} X_{\widetilde{k}}=0$.

(2) Let $p \neq 3$ be a prime number and let $k$ be an imaginary quadratic field. If the class number $h_{k}$ of $k$ is not divided by $p$, then $\operatorname{Tor}_{\Lambda_{\tilde{k} / k}} X_{\widetilde{k}}=0$.

These results were shown by observing the base field $k$ and the cyclotomic $\mathbb{Z}_{p^{-}}$ extension $k_{\infty}$ of $k$. When $p=3$ and the completion of $k$ at a prime lying above 3 contains the group of all 3 -rd roots of unity $\mu_{3}$, the fields $k$ and $k_{\infty}$ do not know whether $\operatorname{Tor}_{\Lambda_{\tilde{k} / k}} X_{\widetilde{k}}=0$ or not. In this article, we shall show that $\operatorname{Tor}_{\Lambda_{\tilde{k} / k}} X_{\widetilde{k}} \neq 0$ in this exceptional case.

Theorem 1. Let $p=3$ and let $k=\mathbb{Q}(\sqrt{-m})$ be an imaginary quadratic field with a positive square-free integer $m$ such that $3 \neq m \equiv 3 \bmod 9$. If $3 \nmid h_{k}$, then $\operatorname{Tor}_{\Lambda_{\tilde{k} / k}} X_{\widetilde{k}} \neq 0$.

Received by the editors August 9, 2006 and, in revised form, December 7, 2006.

2000 Mathematics Subject Classification. Primary 11R23.

(C)2007 American Mathematical Society Reverts to public domain 28 years from publication 
One can show that if $k=\mathbb{Q}\left(\mu_{3}\right)$ or if $3 \nmid h_{k}$ and $m \not \equiv 3 \bmod 9$, then $X_{\widetilde{k}} \simeq \Lambda_{\widetilde{k} / k}$, in particular, $\operatorname{Tor}_{\tilde{k} / k} X_{\widetilde{k}}=0$, for the prime 3 . Indeed, these $k$ 's are 3-rational with $\operatorname{Gal}(\widetilde{k} / k) \simeq \mathbb{Z}_{3}^{\oplus 2}$ (see Example 3 of [13]). If $k$ is 3-rational and $\operatorname{Gal}(\widetilde{k} / k) \simeq \mathbb{Z}_{3}^{\oplus 2}$, then $X_{\widetilde{k}} \simeq \Lambda_{\tilde{k} / k}$ by Theorem 2 of [8]. See also the proof of Theorem 1.1 of [4]. Hence we have classified the non-triviality of $\operatorname{Tor}_{\Lambda_{\tilde{k} / k}} X_{\widetilde{k}}$ under the assumption that $p \nmid h_{k}$. As a corollary to Theorem 1 and a result of Davenport and Heilbronn [2, [3] as extended by Horie and Nakagawa [7], we find that there exist infinitely many imaginary quadratic fields $k$ with positive density such that $\operatorname{Tor}_{\Lambda_{\tilde{k} / k}} X_{\widetilde{k}} \neq 0$ for the prime 3.

Corollary 1. Let $\# S$ denote the cardinality of a set $S$. Let $p=3$ and let $\Delta_{k}$ be the discriminant of a number field $k$. Let $K^{-}(x)$ be the set of imaginary quadratic fields $k$ such that $\left|\Delta_{k}\right|<x$ and put $K^{-}(x, 6,9)=\left\{k \in K^{-}(x) \mid \Delta_{k} \equiv 6 \bmod 9\right\}$. Then we have

$$
\liminf _{x \rightarrow \infty} \frac{\#\left\{k \in K^{-}(x, 6,9) \mid \operatorname{Tor}_{\Lambda_{\tilde{k} / k}} X_{\tilde{k}} \neq 0\right\}}{\# K^{-}(x, 6,9)} \geq \frac{1}{2}
$$

and

$$
\liminf _{x \rightarrow \infty} \frac{\#\left\{k \in K^{-}(x, 6,9) \mid \operatorname{Tor}_{\Lambda_{\tilde{k} / k}} X_{\widetilde{k}} \neq 0\right\}}{\# K^{-}(x)} \geq \frac{1}{16} .
$$

(Note that if $3 \neq m \equiv 3 \bmod 9$, then $\Delta_{\mathbb{Q}(\sqrt{-m})} \equiv 6 \bmod 9$.)

Corollary 1 is shown by the inequality

$$
\#\left\{k \in K^{-}(x, 6,9) \mid \operatorname{Tor}_{\Lambda_{\tilde{k} / k}} X_{\widetilde{k}} \neq 0\right\} \geq \#\left\{k \in K^{-}(x, 6,9) \mid 3 \nmid h_{k}\right\}
$$

and doing estimation similar to the proof of a result of Taya (Theorem 2 of [16]). His description is very easy to understand.

Furthermore, we have an application on the unramified Iwasawa modules. Let $Y_{F}=\operatorname{Gal}\left(L_{F} / F\right)$ be the Galois group of the maximal unramified pro- $p$ abelian extension $L_{F} / F$ of a number field $F$. It is conjectured by Greenberg that $Y_{\widetilde{k}}$ is a pseudo-null $\Lambda_{\tilde{k} / k}$-module, i.e., there are two relatively prime annihilators, for any finite extension $k / \mathbb{Q}$ (Conjecture 3.5 of [6]). Several works on this conjecture have been done for imaginary quadratic fields and imaginary biquadratic fields (see [1], 9] and [12), and no counterexample has been found yet. As a corollary to Theorem 1, we obtain the failure of the pseudo-nullity for a $\mathbb{Z}_{3}^{\oplus 2}$-extension of certain biquadratic imaginary fields.

Corollary 2. Let $p=3$ and let $k=\mathbb{Q}(\sqrt{-m})$ be an imaginary quadratic field with a positive square-free integer $m$ such that $3 \neq m \equiv 3 \bmod 9$. Then $Y_{\widetilde{k}\left(\mu_{3}\right)}$ is not a pseudo-null $\Lambda_{\widetilde{k}\left(\mu_{3}\right) / k\left(\mu_{3}\right)}$-module, if $3 \nmid h_{k}$.

Corollary 2 immediately follows from Theorem 1 of this article and Proposition 3.6 of [11. Note that the failure of the pseudo-nullity of $Y_{\widetilde{k}\left(\mu_{3}\right)}$ does not contradict Greenberg's conjecture since $\widetilde{k}\left(\mu_{3}\right) \subsetneq \widetilde{k\left(\mu_{3}\right)}$ by the isomorphism (11), and note that $k\left(\mu_{3}\right)$ is not contained in Bandini's results [1. The pseudo-nullity of $Y_{\overline{k\left(\mu_{3}\right)}}$ in this case seems quite mysterious.

Here, we shall set the notation of this article. Let $\widetilde{M}_{k} / k$ be the maximal pro-3 extension unramified outside all primes lying above 3 (resp. maximal pro-3 extension) of an algebraic extension $k$ of $\mathbb{Q}\left(\right.$ resp. $\left.\mathbb{Q}_{3}\right)$, and let $G_{k}=\operatorname{Gal}\left(\widetilde{M}_{k} / k\right)$ be 
its Galois group. Then $M_{k}$ is also defined to be the maximal abelian subfield of $\widetilde{M}_{k}$ and we then have $X_{k}=G_{k}^{\mathrm{ab}}$, where the symbol ab means the maximal pro-3 abelian quotient. If $K / k$ is a pro-3 extension, then the complete group ring $\Lambda_{K / k}=$ $\mathbb{Z}_{3}[[\operatorname{Gal}(K / k)]]$ acts on $X_{K}$ via the inner automorphism. Let $\langle J\rangle \simeq \mathbb{Z} / 2$ and let $M$ be a $\mathbb{Z}_{3}[\langle J\rangle]$-module. Then we have the eigen spaces $M^{ \pm}=\{x \in M \mid J x= \pm x\}$ of $M$ and the decomposition $M=M^{+} \oplus M^{-}$. For any topological group $G$, let $G^{\prime}$ be the topological commutator group of $G$.

\section{Proof of Theorem 1}

Put $K=\mathbb{Q}_{3}\left(\mu_{3}\right)$ and let $M_{K} / K$ be the maximal abelian pro-3 extension. Then, by class field theory, we have an isomorphism

$$
K^{\times} \otimes \mathbb{Z}_{3} \simeq \operatorname{Gal}\left(M_{K} / K\right), x \otimes \alpha \mapsto\left(x, M_{K} / K\right)^{\alpha},
$$

where $\left(, M_{K} / K\right)$ is the reciprocity map.

Put $\langle J\rangle=\operatorname{Gal}\left(K / \mathbb{Q}_{3}\right)$. Then $J$ acts on both groups in (2) and the reciprocity map commutes with the action of $J$. Put $U=\left\{x \in K^{\times} \mid x \equiv 1 \bmod 3\right\}$. Since $\sqrt{-3}$ is a prime element of $K$, one sees that

$$
K^{\times} \otimes \mathbb{Z}_{3}=\mu_{3} \oplus 3^{\mathbb{Z}_{3}} \oplus U \text {. }
$$

Then

$$
\left(K^{\times} \otimes \mathbb{Z}_{3}\right)^{+}=3^{\mathbb{Z}_{3}} \oplus U^{+} \simeq \mathbb{Z}_{3}^{\oplus 2}
$$

and

$$
\left(K^{\times} \otimes \mathbb{Z}_{3}\right)^{-}=\mu_{3} \oplus U^{-} \simeq \mathbb{Z} / 3 \mathbb{Z} \oplus \mathbb{Z}_{3}
$$

as $\mathbb{Z}_{3}$-modules. This shows that there is a unique $\mathbb{Z}_{3}$-extension $K_{\infty}^{a}$ of $K$ such that $K_{\infty}^{a} / \mathbb{Q}_{3}$ is a Galois extension and such that $J$ acts on $\operatorname{Gal}\left(K_{\infty}^{a} / K\right)$ as -1 . Let $K_{\infty} / K$ be the cyclotomic $\mathbb{Z}_{3}$-extension, which is obtained by adjoining the group of all 3-power-th roots of unity $\mu_{3^{\infty}}$. Put $K^{(2)}=K_{\infty} K_{\infty}^{a}$. Then $K^{(2)} / K$ is a unique $\mathbb{Z}_{3}^{\oplus 2}$-extension such that $K^{(2)} / \mathbb{Q}_{3}$ is a Galois extension, $K^{(2)}$ contains $K_{\infty}$ and such that $\operatorname{Gal}\left(K^{(2)} / K\right)^{ \pm} \simeq \mathbb{Z}_{3}$ as $\mathbb{Z}_{3}$-modules. Note that $K^{(2)}$ is the fixed field of $\left(\mu_{3} \oplus 3^{\mathbb{Z}_{3}}, M_{K} / K\right)$.

Let $\Gamma=\operatorname{Gal}\left(K^{(2)} / K\right)$. We then define a subgroup $H_{K^{(2)}}$ of $G_{K}$ to be the kernel of the natural morphism $G_{K} \rightarrow \Gamma$. Let $\gamma_{c}, \gamma_{a} \in G_{K}$ be lifts of a system of topological generators of $\Gamma \simeq \mathbb{Z}_{3}^{\oplus 2}$. Here we note that $X_{K^{(2)}}=H_{K^{(2)}}^{\mathrm{ab}}$. In fact, since

$K \subseteq K^{(2)} \subseteq \widetilde{M}_{K}$, we sees that $\widetilde{M}_{K^{(2)}}=\widetilde{M}_{K}$. In particular, $M_{K^{(2)}}$ is the maximal abelian subextension of $\widetilde{M}_{K} / K^{(2)}$. We want to know the structure of $X_{K^{(2)}}$ as a $\Lambda:=\mathbb{Z}_{3}[[\Gamma]]$-module.

Lemma 1. There exist $\mathfrak{f}, \zeta \in H_{K^{(2)}}$ such that

$$
\left(3, M_{K} / K\right)=\mathfrak{f} \bmod G_{K}^{\prime}
$$

and

$$
\left(\frac{-1+\sqrt{-3}}{2}, M_{K} / K\right)=\zeta \bmod G_{K}^{\prime},
$$

and such that

$$
X_{K^{(2)}}=\Lambda\left[\gamma_{c}, \gamma_{a}\right] H_{K^{(2)}}^{\prime}+\Lambda \mathfrak{f} H_{K^{(2)}}^{\prime}+\Lambda \zeta H_{K^{(2)}}^{\prime},
$$

where we let $\left[\gamma_{c}, \gamma_{a}\right]=\gamma_{c} \gamma_{a} \gamma_{c}^{-1} \gamma_{a}^{-1}$. Furthermore, we have

$$
X_{K^{(2)}}=\Lambda\left[\gamma_{c}, \gamma_{a}\right] H_{K^{(2)}}^{\prime} \oplus\left(\Lambda \mathfrak{f} H_{K^{(2)}}^{\prime}+\Lambda \zeta H_{K^{(2)}}^{\prime}\right), \Lambda\left[\gamma_{c}, \gamma_{a}\right] H_{K^{(2)}}^{\prime} \simeq \Lambda
$$

as $\Lambda$-modules. 
Proof. First, we shall introduce the five-term exact sequence

$$
0 \rightarrow \mathrm{H}^{1}\left(G / H, A^{H}\right) \rightarrow \mathrm{H}^{1}(G, A) \rightarrow \mathrm{H}^{1}(H, A)^{G / H} \rightarrow \mathrm{H}^{2}\left(G / H, A^{H}\right) \rightarrow \mathrm{H}^{2}(G, A) .
$$

Here $G, H$ and $A$ stand for a profinite group, a closed normal subgroup of $G$ and a discrete $G$-module, respectively. For reference, see some books about the Galois cohomology, for example, chapter $1, \S 6$, of [14. By applying the dual of the fiveterm sequence with coefficients in $\mathbb{Q}_{3} / \mathbb{Z}_{3}$ to the exact sequence $1 \rightarrow H_{K^{(2)}} \rightarrow G_{K} \rightarrow$ $\Gamma \rightarrow 1$, the sequence

$$
\mathrm{H}_{2}\left(\Gamma, \mathbb{Z}_{3}\right) \longrightarrow\left(X_{K^{(2)}}\right)_{\Gamma} \longrightarrow G_{K}^{\mathrm{ab}} \longrightarrow \Gamma \longrightarrow 0
$$

is exact. From the fact that $\operatorname{Ker}\left(G_{K}^{\mathrm{ab}} \rightarrow \Gamma\right)=\left(\mu_{3} \oplus 3^{\mathbb{Z}_{3}}, M_{K} / K\right)$ and the above exact sequence, there exist $\mathfrak{f}, \zeta \in H_{K^{(2)}}$ such that $\left(3, M_{K} / K\right)=\mathfrak{f} \bmod G_{K}^{\prime}$ and $\left(\frac{-1+\sqrt{-3}}{2}, M_{K} / K\right)=\zeta \bmod G_{K}^{\prime}$. Let $\mathcal{G}=\left\langle\widetilde{\gamma}_{a}, \widetilde{\gamma}_{c}\right\rangle$ be a free pro-3 group of rank 2 and $1 \rightarrow \mathcal{H} \rightarrow \mathcal{G} \rightarrow \Gamma \rightarrow 1$ a minimal presentation of $\Gamma$ by $\mathcal{G}$ with $\widetilde{\gamma}_{a} \mapsto$ $\left.\gamma_{a}\right|_{K^{(2)}},\left.\widetilde{\gamma}_{c} \mapsto \gamma_{c}\right|_{K^{(2)}}$. Then $\mathcal{H}$ is generated by the commutator $\left[\widetilde{\gamma}_{a}, \widetilde{\gamma}_{c}\right]$ as a closed normal subgroup of $\mathcal{G}$. Let $\mathcal{G} \rightarrow G_{K}$ be a morphism defined by $\widetilde{\gamma}_{a} \mapsto \gamma_{a}, \widetilde{\gamma}_{c} \mapsto \gamma_{c}$. Then we obtain the exact commutative diagram

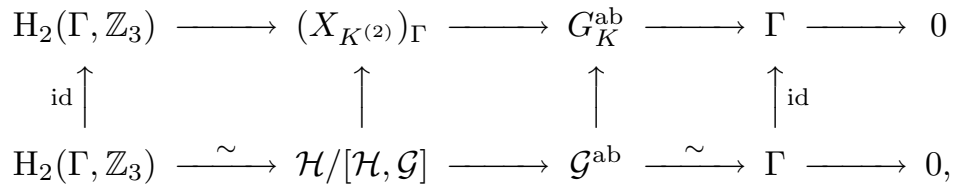

since $\mathcal{G}^{\mathrm{ab}} \simeq \Gamma$ and $\mathrm{H}_{2}\left(\mathcal{G}, \mathbb{Z}_{3}\right)=0$ because $\mathcal{G}$ is a free pro-3 group of rank 2 . It follows that

$$
\begin{aligned}
\operatorname{Image}\left(\mathrm{H}_{2}\left(\Gamma, \mathbb{Z}_{3}\right) \rightarrow\left(X_{K^{(2)}}\right)_{\Gamma}\right) & =\operatorname{Image}\left(\mathcal{H} /[\mathcal{H}, \mathcal{G}] \rightarrow\left(X_{K^{(2)}}\right)_{\Gamma}\right) \\
& =\left[\gamma_{c}, \gamma_{a}\right]^{\mathbb{Z}_{3}}\left[H_{K^{(2)}}, G_{K}\right] /\left[H_{K^{(2)}}, G_{K}\right]
\end{aligned}
$$

Combining the above, by Nakayama's lemma, we have

$$
X_{K^{(2)}}=\Lambda\left[\gamma_{c}, \gamma_{a}\right] H_{K^{(2)}}^{\prime}+\Lambda \mathfrak{f} H_{K^{(2)}}^{\prime}+\Lambda \zeta H_{K^{(2)}}^{\prime} .
$$

Thus the first assertion follows.

Since $h_{\mathbb{Q}\left(\mu_{3}\right)}=1$, the extension $\widetilde{M}_{\mathbb{Q}\left(\mu_{3}\right)} / \mathbb{Q}$ is totally ramified at 3 . Thus

$$
\operatorname{Gal}\left(\widetilde{M}_{\mathbb{Q}\left(\mu_{3}\right)} / \mathbb{Q}\right) \simeq \operatorname{Gal}\left(\mathbb{Q}_{3} \widetilde{M}_{\mathbb{Q}\left(\mu_{3}\right)} / \mathbb{Q}_{3}\right)
$$

Note that there is a unique $\mathbb{Z}_{3}^{\oplus 2}$-extension $\widehat{\mathbb{Q}\left(\mu_{3}\right)} / \mathbb{Q}\left(\mu_{3}\right)$ by the isomorphism (11), which is the compositum of all $\mathbb{Z}_{3}$-extensions of $\mathbb{Q}\left(\mu_{3}\right)$. By the natural morphism, we may identify $\langle J\rangle=\operatorname{Gal}\left(K / \mathbb{Q}_{3}\right)$ with $\operatorname{Gal}\left(\mathbb{Q}\left(\mu_{3}\right) / \mathbb{Q}\right)$. Then one sees that $\operatorname{Gal}\left(\widehat{\mathbb{Q}\left(\mu_{3}\right)} / \mathbb{Q}\left(\mu_{3}\right)\right)^{ \pm} \simeq \mathbb{Z}_{3}$ as $\mathbb{Z}_{3}$-modules. This shows that $K^{(2)}=K \widehat{\mathbb{Q}\left(\mu_{3}\right)}$, whence we can identify $\Gamma$ with $\operatorname{Gal}\left(\widehat{\mathbb{Q}\left(\mu_{3}\right)} / \mathbb{Q}\left(\mu_{3}\right)\right)$. Since $G_{\mathbb{Q}\left(\mu_{3}\right)}$ is a free pro-3 group of rank $2\left(\mathbb{Q}\left(\mu_{3}\right)\right.$ is 3-rational), we find that $G_{\mathbb{Q}\left(\mu_{3}\right)}$ is generated by the images $\left.\gamma_{c}\right|_{\widetilde{M}_{\mathbb{Q}\left(\mu_{3}\right)}}$ and $\left.\gamma_{a}\right|_{\widetilde{M}_{\mathbb{Q}\left(\mu_{3}\right)}}$ of $\gamma_{c}$ and $\gamma_{a}$. Put $H_{\widetilde{\mathbb{Q}\left(\mu_{3}\right)}}=\operatorname{Gal}\left(\widetilde{M}_{\mathbb{Q}\left(\mu_{3}\right)} / \widetilde{\mathbb{Q}\left(\mu_{3}\right)}\right)$. Since $X_{\widetilde{\mathbb{Q}\left(\mu_{3}\right)}}=H_{\widetilde{\mathbb{Q}\left(\mu_{3}\right)}}^{\mathrm{ab}}$ by the same reason mentioned before Lemma 1 , we then have



By observing the natural surjective morphism $X_{K^{(2)}} \rightarrow X_{\widetilde{\mathbb{Q}\left(\mu_{3}\right)}}$, we can conclude that $\Lambda\left[\gamma_{c}, \gamma_{a}\right] H_{K^{(2)}}^{\prime} \simeq \Lambda$ and $X_{K^{(2)}}=\Lambda\left[\gamma_{c}, \gamma_{a}\right] H_{K^{(2)}}^{\prime} \oplus\left(\Lambda \mathfrak{f} H_{K^{(2)}}^{\prime}+\Lambda \zeta H_{K^{(2)}}^{\prime}\right)$. 
Let $k=\mathbb{Q}(\sqrt{-m})$ with $3 \neq m \equiv 3 \bmod 9$. Then one can easily see that the completion of $k$ at the prime above 3 is $K$. Similarly to $\mathbb{Q}\left(\mu_{3}\right)$, we find that $K^{(2)}=K \widetilde{k}$, whence we identify $\Gamma$ and $\Lambda_{\tilde{k} / k}$ with $\operatorname{Gal}(\widetilde{k} / k)$ and $\Lambda$, respectively.

Suppose that $3 \nmid h_{k}$. Then the natural morphism $G_{K} \rightarrow G_{k}$ is surjective. It follows from the facts that $k$ does not contain $\mu_{3}$ and that the unit group of $k$ is finite that there is an isomorphism

$$
\mu_{3} \oplus U \simeq G_{k}^{\mathrm{ab}}
$$

by class field theory. Hence, if it is necessary, we may assume $\mathfrak{f}$ to be in $\mathcal{K}=$ $\operatorname{Ker}\left(G_{K} \rightarrow G_{k}\right)$. Indeed, since $\mathrm{H}_{2}\left(G_{k}, \mathbb{Z}_{3}\right)=0$, we then have the following exact sequence:

$$
0 \longrightarrow \mathcal{K} /\left[\mathcal{K}, G_{K}\right] \longrightarrow G_{K}^{\mathrm{ab}} \longrightarrow G_{k}^{\mathrm{ab}} \longrightarrow 0 .
$$

By the isomorphisms (2) and (4), we find that there is $\mathfrak{f}^{\prime} \in \mathcal{K}$ such that $\mathfrak{f}^{\prime} \bmod G_{K}^{\prime}=$ $\left(3, M_{K} / K\right)$ and that $\mathcal{K}$ is generated by $\mathfrak{f}^{\prime}$ as a closed normal subgroup of $G_{K}$. Since $\mathfrak{f}^{\prime} \in H_{K^{(2)}}(\supseteq \mathcal{K})$ and $\mathfrak{f} \equiv \mathfrak{f}^{\prime} \bmod G_{K}^{\prime}$, we may assume $\mathfrak{f}^{\prime}=\mathfrak{f}$.

Put $H_{\widetilde{k}}=\operatorname{Ker}\left(G_{k} \rightarrow \Gamma\right)$, so that $X_{\widetilde{k}}=H_{\widetilde{k}}^{\text {ab }}$ as mentioned before. Because $\mathrm{H}_{2}\left(G_{k}, \mathbb{Z}_{3}\right)=0$, one sees that $\mathrm{H}_{2}\left(H_{\widetilde{k}}, \mathbb{Z}_{3}\right)=0$. Note that the sequence $1 \rightarrow \mathcal{K} \rightarrow$ $H_{K^{(2)}} \rightarrow H_{\widetilde{k}} \rightarrow 1$ is exact. It follows that $\mathcal{K} /\left[\mathcal{K}, H_{K^{(2)}}\right] \simeq \operatorname{Ker}\left(X_{K^{(2)}} \rightarrow X_{\widetilde{k}}\right)=$ $\Lambda \mathfrak{f} H_{K^{(2)}}^{\prime}$. Here we need the following two results:

Theorem B (Proposition 2.6 of [15] and Example 5.2(c) of [10]). Let $p$ be a prime number, $E / \mathbb{Q}_{p}$ a finite extension and $E^{(d)} / E$ a $\mathbb{Z}_{p}^{\oplus d}$-extension. Then the following two assertions hold:

(1) $\operatorname{rank}_{\Lambda_{E}^{(d)} / E} X_{E^{(d)}}=\left[E: \mathbb{Q}_{p}\right]$.

(2) If $\mu_{p^{\infty}} \subseteq E^{(d)}$ and if $\operatorname{Gal}\left(E^{(d)} / E\right) \simeq \mathbb{Z}_{p}^{\oplus 2}$, then $X_{E^{(d)}}$ has no non-trivial $\Lambda_{E^{(d)} / E^{-} \text {-torsion. }}$

Property (2) of Theorem B is deduced from the explicit structure of $X_{K^{(2)}}$ as a $\Lambda$-module given in [10. What we did in this article was to argue the structure with given generators of $X_{K^{(2)}}$.

From Theorem B, we find that $\operatorname{rank}_{\Lambda} X_{K^{(2)}}=2$, whence

$$
\operatorname{rank}_{\Lambda}\left(\Lambda \mathfrak{f} H_{K^{(2)}}^{\prime}+\Lambda \zeta H_{K^{(2)}}^{\prime}\right)=1 .
$$

Furthermore, since $X_{K^{(2)}}$ has no $\Lambda$-torsion, we get $\Lambda \mathfrak{f} H_{K^{(2)}}^{\prime} \simeq \Lambda$. This shows that

$$
\left(\Lambda \mathfrak{f} H_{K^{(2)}}^{\prime}+\Lambda \zeta H_{K^{(2)}}^{\prime}\right) / \Lambda \mathfrak{f} H_{K^{(2)}}^{\prime}
$$

is a non-trivial torsion $\Lambda$-submodule of $X_{\widetilde{k}}$. This completes the proof of Theorem 1.

\section{ACKNOWLEDGMENTS}

The author would like to express his thanks to Professor Ken Ono for enlightening him about the results of Davenport-Heilbronn [2], 3] and Horie-Nakagawa [7, pointing out Corollary 1 and giving a valuable suggestion. The author would also like to express his thanks to Hisao Taya for pointing out his paper [16. The author would also like to express his thanks to the referee for pointing out the second inequality of Corollary 1 and giving valuable advice. 


\section{REFERENCES}

[1] A. Bandini, Greenberg's conjecture for $\mathbb{Z}_{p}^{d}$-extensions, Acta Arith. 108 (2003), 357-368. MR1979904 (2004c:11201)

[2] H. Davenport and H. Heilbronn, On the density of discriminants of cubic fields, Bull. London Math. Soc. 1 (1969), 345-348. MR0254010 (40:7223)

[3] H. Davenport and H. Heilbronn, On the density of discriminants of cubic fields. II, Proc. Roy. Soc. London Ser. A 322 (1971), 1551, 405-420. MR0491593 (58:10816)

[4] S. Fujii, The torsion of $p$-ramified Iwasawa modules, submitted.

[5] R. Greenberg, On the structure of certain Galois groups, Invent. Math. 47 (1978), 85-99. MR.504453 (80b:12007)

[6] R. Greenberg, Iwasawa theory-past and present, Class field theory-its centenary and prospect (Tokyo, 1998), 335-385, Adv. Stud. Pure Math., 30, Math. Soc. Japan, Tokyo, 2001. MR1846466 (2002f:11152)

[7] K. Horie and J. Nakagawa, Elliptic curves with no rational points, Proc. Amer. Math. Soc. 104 (1988), 20-24. MR958035 (89k:11113)

[8] Y. Ihara, Profinite braid groups, Galois representations and complex multiplications, Ann. Math. 123 (1986), 43-106. MR825839 (87c:11055)

[9] T. Itoh, Pseudo-null Iwasawa modules for $\mathbb{Z}_{2}^{2}$-extensions, submitted for publication.

[10] U. Jannsen, Iwasawa modules up to isomorphism, Algebraic number theory, 171-207, Adv. Stud. Pure Math., 17, Academic Press, Boston, MA, 1989. MR1097615 (93c:11095)

[11] A. Lannuzel and T. Nguyen Quang Do, Conjectures de Greenberg et extensions pro- $p$-libres d'un corps de nombres, Manuscripta Math. 102 (2000), 187-209. MR1771439(2001e:11106)

[12] J. Minardi, Iwasawa modules for $\mathbb{Z}_{p}^{d}$-extensions of algebraic number fields, Thesis (1986), Washington University.

[13] A. Movahhedi, Sur les p-extensions des corps p-rationneles, Math. Nachr. 149 (1990), 163176. MR.1124802(92j:11131)

[14] J. Neukirch, A. Schmidt and K. Wingberg, Cohomology of number fields, Grundlehren der Mathematischen Wissenschaften, 323, Springer-Verlag, Berlin, 2000. MR1737196 (2000j:11168)

[15] T. Nguyen Quang Do, Formations de classes et modules d'Iwasawa, Lecture Notes in Math., 1068, Springer, Berlin, 1984, 167-185. MR756093 (85j:11156)

[16] H. Taya, Iwasawa invariants and class numbers of quadratic fields for the prime 3, Proc. Amer. Math. Soc. 128 (2000), 1285-1292. MR1641133 (2000j:11162)

[17] L. C. Washington, Introduction to cyclotomic fields. Second edition, Graduate Texts in Mathematics, 83. Springer-Verlag, New York, 1997. MR1421575 (97h:11130)

Department of Mathematical Sciences, Graduate School of Science and Engineering, Keio University, Hiyoshi, Kohoku-Ku, Yokohama City, Kanagawa, 223-8522, Japan

E-mail address: moph@a2.keio.jp 